\title{
Spontaneous bacterial peritonitis in cystic fibrosis
}

\author{
C F Doershuk, R C Stern
}

\begin{abstract}
Bacterial peritonitis presents with classic symptoms of fever and abdominal pain. Some patients, however, are completely asymptomatic. Death in the short term is considerable, especially in patients with alcoholic cirrhosis. Cystic fibrosis patients occasionally develop biliary cirrhosis and may have secondary hypersplenism, varices, and ascites. These patients should be at risk for spontaneous bacterial peritonitis. Spontaneous bacterial peritonitis is described in two patients with longstanding hepatic cirrhosis secondary to cystic fibrosis. Both had required splenectomy for complications of portal hypertension. This is a previously unreported, but potentially fatal, complication of cystic fibrosis liver disease. Early diagnostic paracentesis is essential so that appropriate acute management, including antimicrobial treatment can be started. In the long term, these patients deserve immediate paracentesis for any evidence of recurrence. Whether the patient is treated with chronic (continuous) antimicrobial prophylaxis or only receives antimicrobial treatment during periods when bacteraemia is possible (for example, dental work, bronchoscopy), it would seem reasonable in patients with cystic fibrosis to use a wide spectrum antimicrobial agent with activity against Pseudomonas aeruginosa, other common Gram negative organisms, and Stpahylococcus aureus.

(Gut 1994; 35: 709-711)
\end{abstract}

In 1958, Caroli and Platteborse reported on a series of patients with cirrhosis with coliform septicaemia or peritonitis complicating ascities, or both. ${ }^{1}$ Two more reports in the 1960 s confirmed that this syndrome was more common than generally recognised and was often misdiagnosed. ${ }^{23}$ Gram negative organisms, mainly Escherichia coli and Klebsiella sp, are most commonly encountered; however, Gram positive cocci, including streptococcus, pneumococcus, and staphylococcus, have been reported, ${ }^{4-8}$ suggesting both bowel and haematological sources for the infection. In a review of published works and summary of their experience with 28 patients with alcoholic cirrhosis, Conn and Fessel introduced the term spontaneous bacterial peritonitis (SBP). They noted a very high inhospital mortality, suggested that ascites was the sine qua non of the syndrome, and described a wider variety of clinical features than previously appreciated. They also extended the syndrome to include patients without bacteraemia. ${ }^{8}$ SBP is an ascitic infection without a local contiguous source, such as abscess or intestinal perforation. ${ }^{9}$ After an additional report of culture negative, but probable SPB, ${ }^{10}$ Runyon and Hoefs reported 18 episodes of culture negative neutrocytic ascites, ${ }^{11}$ with many similarities to SBP. Subsequently, SBP and culture negative neutrocytic asites have been reported in many conditions. ${ }^{12}$ Patients with alcoholic cirrhosis and peritonitis have substantial inhospital mortality, ${ }^{38}$ with some improvement after earlier diagnosis and improved antimicrobial treatment.?

Patients with cystic fibrosis may have biliary cirrhosis with portal hyertension and ascites. ${ }^{13}$ Although they would be expected to be at risk, to our knowledge SBP has not been reported in cystic fibrosis. We report here two cystic fibrosis patients, ages 21 and 36 years with known liver disease, who developed this complication of ascites.

\section{Case reports}

Eight of 1310 cystic fibrosis patients seen at this centre over a 35 year period have had a splenectomy. Two of these patients had no portal systemic shunting procedure, and both subsequently have developed SBP.

\section{CASE 1}

Cystic fibrosis presented at birth with meconium ileus. At age 13, acute biliary colic led to a diagnosis of cholelithiasis, and a cholecystectomy was performed. Open liver biopsy showed changes consistent with cystic fibrosis related cirrhosis. The patient stayed in hospital only twice during her teens for intravenous antimicrobial treatment of pulmonary exacerbations. She gradually developed, however, massive splenomegaly, severe splenic pain, and laboratory evidence of hypersplenism. At age 20, a splenectomy was necessary, but a shunt was not performed because of the possibility of rapid hepatic decompensation and need for liver transplant. Postoperatively, liver function was marginal and the patient's functional state and feeling of well being were very poor. Ascites necessitated aggressive diuretic treatment. She was tentatively listed for liver transplant. At age 21 , she presented with abdominal pain and fever. The peripheral leucocyte count was $9 \cdot 1 \times 10^{9} / 1$ $\left(9100 / \mathrm{mm}^{3}\right)$ with $66 \%$ neutrophils. A paracentesis showed a leucocyte count of $1.5 \times 10^{9} / 1$ (1575/ $\mathrm{mm}^{3}$ ) with $77 \%$ neutrophils. The ascitic fluid glucose was $5.0 \mathrm{mmol} / \mathrm{l}$, the protein $23 \mathrm{~g} / \mathrm{l}$, and the $\mathrm{LDH}(\mathrm{l} \rightarrow \mathrm{P}) 144 \mathrm{U} / \mathrm{l}$; the $\mathrm{pH}$ was not reported. The ascitic fluid culture showed $E$ coli. The patient's sputum culture yielded only $P$ seudomonas aeruginosa on culture; $E$ coli had not been present in respiratory tract secretions. The patient responded to intravenous antimicrobials with resolution of abdominal pain and fever, and improved feeling of well being. Repeat ascitic fluid culture yielded no growth. 
The patient had one recurrence of abdominal pain. Ascitic fluid cultures were negative, but the patient was taking antimicrobials. Subsequently, she continued on antimicrobial prophylaxis (norfloxacin) and has not had a recurrence of abdominal pain for 23 months.

\section{CASE 2}

A 36 year old pharmacist with cystic fibrosis, had a long history of gastrointestinal and pulmonary symptoms. At age 26 years, he had a splenectomy for hypersplenism. The liver was fibrotic and multinodular, consistent with biliary cirrhosis. A shunting procedure could not be performed because the splenic vein was too thin walled and fragile. At 36 years, during a pulmonary exacerbation, the total serum protein was $55 \mathrm{~g} / \mathrm{l}$ and albumin $28 \mathrm{~g} / \mathrm{l}$; bilirubin total $46 \cdot 2$ $\mu \mathrm{nol} / \mathrm{l}$ and direct $29 \cdot 1 \mu \mathrm{g} / \mathrm{l}$, alkaline phosphatase $237 \mathrm{U} / \mathrm{l}$, serum aspartate aminotransferase (AST), $1170 \mathrm{nmol} / \mathrm{sec}^{-1} / 1$, serum alanine aminotransferase (ALT) $1173 \mathrm{nmol} / \mathrm{sec}^{-1} / 1$, and $\gamma$-glutamyl-transferase $167 \mathrm{U} / 1$. He had no ascites, He returned to full time work. Seven months after, he developed abdominal swelling, mild peripheral oedema, and some dyspnoea, all unresponsive to salt restriction. Abdominal swelling increased, and an ultrasound at another centre confirmed massive ascites, which they attributed to an albumin of $24 \mathrm{~g} / \mathrm{l}$. He was in hospital elsewhere but discharged two days later despite low grade fever, moderate diffuse abdominal pain, and an increasing white blood count $\left(8.8 \times 10^{9} / 1\right.$ to $12.7 \times 10^{9} / 1$. He presented at our centre the next day. Paracentesis yielded 700 ml of cloudy fluid with a leucocyte count of $6 \cdot 27 \times 10^{9} / 1(80 \%$ neutrophils $)$, glucose $8.1 \mathrm{mmol} /$ 1 , protein $4.0 \mathrm{~g} / \mathrm{l}$. Cultures for fungi, mycobacteria, viruses, and repeated bacterial cultures were all negative; however, he had received oral ciprofloxacin for several days. Total serum protein at admission was $50 \mathrm{~g} / 1$, and the albumin $19 \mathrm{~g} / \mathrm{l}$. Alkaline phosphatase $275 \mathrm{U} / 1$, AST 2122 $\mathrm{nmol} / \mathrm{sec}^{-1}-1$, ALT $1207 \mathrm{nmol} / \mathrm{sec}^{-1} / 1$, and $\gamma-$ glutamyltransferase $211 \mathrm{U} / 1$. Total and direct bilirubin were $13.68 \mu \mathrm{mol} / 1$ and $3.4 \mu \mathrm{mol} / 1$. Additional paracenteses yielded large amounts (up to $1700 \mathrm{ml}$ ) of cloudly fluid, which remained culture negative. He responded to antimicrobial treatment with disappearance of fever, decrease in white blood count, rise in albumin $22 \mathrm{~g} / \mathrm{l}$, and no reaccumulation of ascites. He was discharged on prophylactic antimicrobial (norfloxacin). He has not had a recurrence of abdominal pain, and at follow up 10 months later, there was minimal ascites.

\section{Discussion}

Up to $10 \%$ of patients with alcoholic cirrhosis and overt portal hypertension with ascites develop SBP. ${ }^{8}$ SBP also complicates other diseases, including nephrotic syndrome, disseminated lupus erythematosus, haemochromatosis, primary biliary cirrhosis, chronic active hepatitis, and acute hepatitis. ${ }^{12}$ Focal biliary cirrhosis has been reported in 5-9\% of cystic fibrosis patients ${ }^{1314}$ with a few progressing to diffuse multilobular involvement. ${ }^{1516}$ Only a small percentage progress to symptomatic portal hypetension, including ascites or clinically significant oesophageal varices, or both. A substantial number $(36 \%)$ have low concentrations of serum albumin $(<25 \mathrm{~g} / \mathrm{l}) .^{16}$

Because bacterial peritonitis may present with minimal or no symptoms ${ }^{710}$ and may be afebrile, ${ }^{9}$ a high index of suspicion is necessary to ensure the early treatment needed to prevent death. Bacterial peritonitis should be excluded by a diagnostic peritoneal tap in any patient with ascites and abdominal pain or fever, or both. ${ }^{10}$ The bedside inoculation of blood culture bottles $^{17}$ decreases the incidence of negative cultures from 40 to $10 \% .^{9}$ The $\mathrm{pH}$ of the ascitic fluid may aid in discriminating between infected ( $\mathrm{pH} 7 \cdot 25(0.06)$, range $7 \cdot 25-7 \cdot 31)$ and uninfected $(7 \cdot 47(0 \cdot 07)$, range $7 \cdot 39-7 \cdot 58)$ ascitic fluid. ${ }^{18}$

There is some variability in the recommendation for antimicrobial treatment. Munoz and Maddrey suggest that intravenous antimicrobial treatment, regardless of Gram stain or culture results, should be started when more than $0.25 \times 10^{9} / 1$ neutrophils are present in ascitic fluid. ${ }^{19}$ Conn and Atterbury propose that antimicrobial treatment should be started whenever the clinical picture of SBP is present, even in the absence of polymorphonuclear leucocytes in the ascitic fluid; whenever the number of polymorphonuclear leucocytes in the ascitic fluid is greater than $0.5 \times 10^{9} / 1$, and the clinical picture is compatible with SBP - that is, unexplained fever, abdominal pain; and whenever the number of neutrophils is greater than $1 \times 10^{9} / 1$, even in the absence of any evidence of SBP. ${ }^{12}$ Because of the increased risk of nephrotoxicity in alcoholic patients with SBP, ${ }^{20}$ some caution in the use of aminoglycosides may also be indicated in cystic fibrosis patients with SBP.

SBP in alcoholic cirrhosis is an ominous event with high inhospital mortality. ${ }^{38}$ One report shows that only a few patients survive three years. ${ }^{7}$ A large series of patients with predominantly non-alcoholic cirrhosis, found SBP to be comparatively common (24\%). ${ }^{10}$ The bacteriological results were similar to those of alcoholic cirrhosis, but the inhospital mortality was lower: $48 \%$ of all the SBP cases, and $22 \%$ if the culture was positive but the neutrophil count was less than $0.25 \times 10^{9} / 1$

Both of our cystic fibrosis patients had biliary cirrhosis and hypersplenism requiring splenectomy. A shunt procedure was possible in the other six of eight patients who required splenectomy. Splenectomy without shunt may put cystic fibrosis patients at greater risk of SBP in the presence of ascites. Early diagnostic paracentesis and prompt antimicrobial treatment led to clearing of symptoms and signs in both patients. Based on clinical experience with patients with alcoholic cirrhosis and peritonitis, both of our patients were given prophylactic antimicrobial treatment and have survived 23 months and 10 months respectively without recurrence. The antimicrobial agent we chose (norfloxacin) is effective against Staphylococcus aureus, as well as common Gram negative organisms, including $P$ aeruginosa.

While peritonitis may recur in alcoholic 
cirrhosis, ${ }^{8}$ there is no reported experience in cystic fibrosis. In the prospective, predominantly non-alcoholic cirrhosis series, ${ }^{10}$ morphological diagnosis was available in only $13 \%$ and there was no follow up information. Depending on the culture and white blood cell findings, these authors found a $50-75 \%$ inhospital survival, which they felt could be attributed to early treatment or less advanced peritonitis, or both. ${ }^{10}$ Depressed phagocytic activity, reduced serum complement concentrations, low opsonin activity in ascitic fluid, impaired leucocyte function $^{2122}$ and low concentrations of serum albumin (less than $30 \mathrm{~g} / \mathrm{l}$ in all 23 patients with a reported albumin concentration $)^{8}$ are reported in alcoholic cirrhosis. There is no comparable information available in cystic fibrosis except that albumin concentrations of $32 \mathrm{~g} / 1$ or greater were noted in 9 of 14 cystic fibrosis patients with symptomatic hepatic disease. ${ }^{16}$ These results may reflect the differing pathophysiology in the two conditions and may have some influence on prognosis or indications, or both for liver transplant.

Conn and Fessel ${ }^{8}$ showed that ascitic cirrhotic patients seem to have as great a risk of infection after dental procedures as do patients with rheumatic valvular disease. They suggested that prophylactic antimicrobial treatment for dental procedures, colonic preparation for sigmoidoscopy or barium enema, and paracentesis might be indicated in these cirrhotic patients. Lacking definitive data and appreciating the high risk of recurrence, we have elected to use continuous prophylasix instead. Furthermore, as our patients with SBP were the only cystic fibrosis patients (out of 1310 patients seen over 35 years) who had a splenectomy without a shunting procedure, it may be reasonable to use prophylactic antimicrobials on all such patients who develop ascites.

In summary, we report two cystic fibrosis patients with cirrhosis who had a splenctomy who developed SBP complicating ascites. Prompt treatment and subsequent antimicrobial prophylaxis was successful and both have survived 23 and 10 months respectively without recurrence. Bacterial peritonitis is a potentially fatal complication of ascites in cirrhotic cystic fibrosis patients. Early diagnosis and intravenous antimicrobial intervention may be as important to short term survival and longterm outcome in cirrhotic cystic fibrosis patients as they are in alcoholic cirrhosis.

Supported in part by Grant DK 27651 from the NIH, United Way Services of Cleveland, and the Cystic Fibrosis Foundation.

1 Caroli J, Platteborse R. Septicemie porto-cave: cirrhoses du foie et septicemie a colibacille. Sem Hop Paris 1958; 34: 47287.

2 Kerr DNS, Pearson DT, Read AE. Infection of ascitic fluid in patients with hepatic cirrhosis. Gut 1963; 4: 394-8.

3 Conn HO. Spontaneous bacterial peritonitis and bacteremia in Laennec's cirrhosis caused by enteric organisms. Ann Intrn Med 1964; 60: 568-80.

4 Ginsberg MD. Spontaneous group B streptococcal bacteria complicating hepatic cirrhosis: report of two cases. Am $\mathcal{F}$ Dig Dis 1968; 13: 1065-71.

5 Epstein M, Calia MD, Gabuzda GJ. Pneumococcal peritonitis in patients with postnecrotic cirrhosis. $N$ Engl f Med 1968; 278: 69-73.

6 Correia JP, Conn HO. Spontaneous bacterial peritonitis in cirrhosis: endemic or epidemic? Symposium on diseases of the liver. Med Clin North Am 1975; 59: 963-83.

7 Hoefs JC, Canawati HN, Sapico FL, Hopkins RR, Weiner J, Montgomerie JZ. Spontaneous bacterial peritonitis. Hepatology 1982; 2: 399-407.

8 Conn HO, Fessel JM. Spontaneous bacterial peritonitis in cirrhosis: variations on a theme. Medicine 1971; 50: 161-97.

9 Hoefs JC. Portal hypertension. In: Gitnick G, ed. Principles and practice of gastroenterology and hepatology. Ist ed. New York: Elsevier, 1988: 1262-6.

10 Pinzello G, Simonetti RG, Craxi A, Di Piazza S, Spano C, Pagliaro L. Spontaneous bacterial peritonitis: a prospective investigation in predominantly nonalcoholic cirrhotic patients. Hepatology 1983; 3: 545-9.

11 Runyon BA, Hoefs JC. Culture-negative neutrocytic ascites: a variant of spontaneous bacterial peritonitis. Hepatology variant of spont $1209-11$.

12 Conn HO, Atterbury CE. Cirrhosis. In: Schiff L, Schiff ER, ed. Diseases of the liver. 6th ed. Philadelphia: JB Lippincott, 1987: 829-64

13 di Sant 'Agnese PA, Davis PB. Cystic fibrosis in adults. $\mathrm{Am} \mathcal{F}$ Med 197.9; 66: 121-32.

14 Craig JM, Haddad H, Shwachman H. The pathological changes in the liver in cystic fibrosis of the pancreas. Am $\mathcal{F}$ Dis Child 1957; 93: 357-69.

15 Shwachman H, Kowalski M, Khaw KT. Cystic fibrosis: a new outlook. Medicine 1977; 56: 129-49.

16 Stern RC, Stevens DP, Boat TF, Doershuk CF, Izant RJ, Matthews LW. Symptomatic hepatic disease in cystic fibrosis: incidence, course and outcome of portal systemic shunting. Gastroenterology 1976; 70: 645-9.

17 Runyon BA, Umland ET, Merlin T. Inoculation of blood culture bottles with ascitic fluid: improved detection of culture bottles with ascitic fluid: improved detection of 147: 73-5.

18 Gitlin N, Stauffer JL, Silvestri RC. The pH of ascitic fluid in the diagnosis of spontaneous bacterial peritonitis in alcoholic cirrhosis. Hepatology 1982; 2: 408-11.

19 Munoz SJ, Maddrey WC. Major complications of acute and chronic liver disease. Gastroenterol Clin North Am 1988; 17: 265-86.

20 Cabrera J, Arroyo V, Ballesta AM, Rimola A, Gaul J, Elena M, et al. Aminoglycoside nephrotoxicity in cirrhosis. Gastroenterology 1982; 82: 97-105.

21 Runyon BA. Low-protein concentration ascitic fluid is predisposed to spontaneous bacterial peritonitis. Gastroenterology 1986; 91: 1343-6.

22 Runyon BA, Van Epps DE. Diuresis of cirrhotic ascites increased its opsonic activity and may help to prevent spontaneous bacterial peritonitis. Hepatology 1986; 6: 396-9. 